\title{
The influence of laboratory-induced MELD score differences on liver allocation: more reality than myth
}

Schouten JN, Francque S, Van Vlierberghe H, Colle I, Nevens F, Delwaide J, Adler M, Starkel P, Ysebaert D, Gadisseur A, De Winter B, Smits JM, Rahmel A, Michielsen P. The influence of laboratory-induced MELD score differences on liver allocation: more reality than myth.

Clin Transplant 2011 DOI: 10.1111/j.1399-0012.2011.01538.x. (C) 2011 John Wiley \& Sons A/S.

Abstract: Background: Liver allocation in Eurotransplant (ET) is based on the MELD score. Interlaboratory MELD score differences in INR and creatinine determination have been reported. The clinical implication of this observation has not been demonstrated.

Methods: MELD scores were calculated in 66 patients with liver cirrhosis using bilirubin, creatinine, and INR analyzed in six liver transplant centers. Based on allocation results of ET, patients transplanted from December 2006 to June 2007 were divided according to MELD score in four groups. For each group, the influence of the match MELD on the probability of receiving a transplant was studied (Cox proportional hazards model).

Results: Laboratory-dependent significant differences in MELD score were demonstrated. Cox proportional hazards model showed a significant association between MELD score and the probability of organ allocation. The unadjusted hazard ratio for receiving a liver transplant was significantly different between group 2 and group 4 (group 2: MELD 19-24; group 4: MELD > 30).

Conclusion: Laboratory-dependent significant differences in MELD score were observed between the six transplant centers. We demonstrated a significant association between the MELD score and the probability of organ allocation. The observed interlaboratory variation might yield a significant difference in organ allocation in patients with high MELD scores.

\author{
J.N. Schouten ${ }^{a}$, S. Francque ${ }^{a}$, \\ H. Van Vlierberghe ${ }^{b}$, I. Colle ${ }^{b}$, \\ F. Nevens ${ }^{c}$, J. Delwaide ${ }^{d}$, \\ M. Adler ${ }^{\mathrm{e}}$, P. Starkel ${ }^{\mathrm{f}}$, D. Ysebaert ${ }^{\mathrm{a}}$, \\ A. Gadisseurg, B. De Winter ${ }^{\mathrm{h}}$, \\ J.M. Smitsi, A. Rahmel' and \\ P. Michielsen ${ }^{\mathrm{a}}$
}

${ }^{a}$ Department of Gastroenterology and Hepatology, University Hospital Antwerp, Antwerp Belgium, ${ }^{\mathrm{b}}$ Department of Gastroenterology and Hepatology, University Hospital Ghent, Ghent, Belgium, 'Department of Hepatology, University Hospital Leuven, Leuven, Belgium, 'Department of Gastroenterology and Hepatology, University Hospital Liège, Liège, Belgium, ${ }^{e}$ Department of Gastroenterology and Hepatology, University Hospital Erasme, Brussels, Belgium, 'Department of Gastroenterology and Hepatology, University Hospital St Luc, Brussels, Belgium, 9Department of Hematology, University Hospital Antwerp,

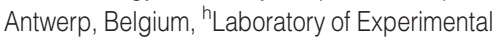
Medicine and Paediatrics, University of Antwerp, Antwerp, Belgium and 'Eurotransplant International Foundation, Leiden, the Netherlands

\footnotetext{
Key words: allocation - bilirubin - creatinine international normalized ratio - liver

transplantation - MELD score

Corresponding author: Jeoffrey Schouten, MD, Department of Gastroenterology Hepatology, Erasmus Medical Center, 's Gravendijkwal 230, 3015 GD Rotterdam, The Netherlands. Tel.: +31 6 12767851; fax: +31 107035172 ; e-mail: j.schouten@erasmusmc.nl

Accepted for publication 21 July 2011
}

In 1999, the Institute of Medicine issued a mandate to the liver transplant community to design a new organ allocation system that would deemphasize waiting time and emphasize severity of liver disease for determining priorities in allocating organs for liver transplantation (LT). In response, the model for end-stage liver disease (MELD) score, origi- nally created to predict survival following elective placement of TIPS (transjugular intrahepatic portosystemic shunt), was proposed as a fair score for organ allocation $(1,2)$. Being a mathematical function including only objective laboratory variables (international normalized ratio [INR], bilirubin, and creatinine), this score outscores the 
previously applied Child score that includes two subjective parameters (the presence of ascites and hepatic encephalopathy) baring the possibility of subjective influence. By including widely available objective laboratory variables, the MELD score "theoretically" is free of subjective bias. The rationale behind using this model is "the sickest first" policy, in which not the time on the waiting list but the mortality risk determines to whom an organ is allocated. Since its implementation, the MELD score has retrospectively and prospectively shown to be highly predictive of short-term mortality in patients with end-stage liver disease awaiting LT (1, 3-7). As a result, the introduction of the MELD score system by UNOS in 2002 (United Network of Organ Sharing) has resulted in a significant reduction in death on the waiting list $3.5 \%$ reduction in waiting list mortality in the United States), while early survival of liver transplant recipients remained unchanged despite the selection of more ill patients $(8,9)$. Based on these data, Eurotransplant (ET), an organization responsible for the mediation and allocation of organ donation procedures in Austria, Belgium, Croatia, Germany, Luxemburg, the Netherlands, and Slovenia, also introduced the MELD score in liver allocation in December 2006.

However, despite the advantages of this allocation system in liver transplantation, the use of the MELD score to prioritize liver transplant recipients is not without limitations. Since its implementation in liver allocation, reports discussing not only strengths, but also limitations, have been published (10-17). An important flaw of this model is that it cannot take into account institutional variation in laboratory methodologies, in the measurement of serum creatinine, bilirubin, and INR. The latter test has only been validated in patients taking oral anticoagulant therapy (OA). The application of INR in other categories of patients (e.g., in liver disease) is scientifically flawed. Trotter et al. (18) were the first to yield the clinical problem resulting from the incorporation of the INR in the MELD. In a study of 29 patients listed for liver transplantation, they demonstrated laboratory methodology-dependent differences in MELD score resulting from differences in INR determination (not so for bilirubin and creatinine). A point of criticism on this study was relative low mean MELD scores in the studied population (center 1: 13.6, center 2: 14.7, center 3: 17.1), whereas patients on the waiting list mostly have MELD scores higher than 17.

Contrasting interlaboratory differences in INR, Trotter et al. did not demonstrate significant differences in bilirubin and creatinine. Conversely, differences in creatinine were reported in patients with or without liver disease (19-21).

Cholongitas et al. (20) showed that the utilization of different laboratory assays for creatinine contributed to significant different MELD scores. The highest MELD scores were observed in the group with the highest bilirubin, because of the negative interference of bilirubin elevation with creatinine measurements. These results match with previously presented data $(19,21)$. Recently, Lisman et al. (22) confirmed this variation in MELD score parameters because of interlaboratory differences (most important for INR) in a large group of patients with liver disease.

Presumably, this laboratory dependency in MELD score calculation might lead to systemic biases possibly influencing prioritization of some patients over others. However, statistically significant differences in the probability of receiving a post-mortem liver transplant because of these laboratory-dependent differences have not been shown yet (23).

We performed a multicenter study to analyze center-dependent variation in creatinine, bilirubin, INR, and MELD score in a large study population with high mean MELD, divided according to Child classification. Different methods, instruments, and thromboplastins were used in the participating centers allowing us to estimate the impact of each of these elements on potentially observed differences. To demonstrate statistically significant differences in the probability of receiving a liver transplant because of laboratory-dependent differences in MELD score, waiting list and liver allocation results from ET for the first six months after MELD implementation were collected.

\section{Patients and methods}

Peripheral blood samples were collected from 66 patients with biopsy-proven cirrhosis (15 Child A, 23 Child B, and 28 Child C) in the Departments of Hepatology and Gastroenterology of the University Hospital Antwerp and the University Hospital Ghent between December 2006 and June 2007. After obtaining informed consent, patients were prospectively enrolled in the study. From each patient, six blood aliquots were collected, each aliquot contained samples for the analysis of creatinine, bilirubin, and INR. Control aliquots from 30 healthy volunteers without evidence of liver failure, renal failure, or hemostasis disorders and 30 patients taking OAs without evidence of liver disease were collected for the calculation of the three parameters of the MELD score. All samples were immediately frozen at minus $20^{\circ} \mathrm{C}$, 
not longer than three months for bilirubin/creatinine, and at minus $80^{\circ}$ for PT/INR. Samples for bilirubin determination were protected from light. Analyses of all samples were performed at the same day in the laboratories of the Belgian liver transplant centers (University Hospital Ghent, University Hospital Leuven, University Hospital Liège, University Hospital Erasme, Brussels, University Hospital St Luc, Brussels, and University Hospital Antwerp). The first aliquot remained in the University Hospital Antwerp or University Hospital Ghent for analyses. The other five were sent (frozen) for analysis to the laboratories of the other five Belgian transplant centers. The specific methods, instruments, and thromboplastins for each essay and hospital are shown in Table 1. Mean values of creatinine, bilirubin, and INR were compared between the six different laboratories.

MELD scores were calculated for each patient with liver cirrhosis using bilirubin, creatinine, and INR retained from the six different laboratories according to the formula currently in use by ET and UNOS [MELD score $=\left(9.57 \times \log _{\mathrm{e}}\right.$ creatinine $\mathrm{mg} / \mathrm{dL}+3.78 \times \log _{\mathrm{e}}$ bilirubin $\mathrm{mg} / \mathrm{dL}+$ $\left.\left.11.20 \times \log _{\mathrm{e}} \mathrm{INR}+6.43\right)\right]$ (2). Patients with a MELD score above 17 in center 2 were selected to study MELD score differences in patients with high scores.

A linear mixed model with a repeated effect controlling for replicated measurements across the six centers for each subject was used to analyze bilirubin, INR, creatinine, and Meld score. The analysis was performed separately for the different patient groups.

Patients $(n=214)$ transplanted in the first six months of MELD score implementation (December 2006-June 2007) were divided according MELD score in four groups (group 1: MELD 11-18; group 2: MELD 19-24; group 3: MELD 25-29; group 4: MELD > 30) based on the ET current practice of duration in a specific MELD class (http://www.eurotransplant.org). For each group, the influence of the match MELD on the probability of receiving a transplant was studied in a Cox proportional hazards model (univariate and multivariate).

\section{Results}

Mean INR for cirrhotic patients, controls, and patients on OAs were compared, and results are shown in Table 2 and Fig. 1A. Laboratories using a thromboplastin with a high International Sensitivity Index (ISI) (laboratories 2, 4, and 6) clearly demonstrated a statistical significant higher INR compared with those using a thromboplastin with a low ISI (laboratories 1, 3, and 5). The highest mean interlaboratory differences were present between laboratory 1 and laboratory 2 (difference in mean INR: 0.42). The interlaboratory INR
Table 1. Instruments, methods, and thromboplastins used in the six participating laboratories for determination of the three parameters of the MELD score

\begin{tabular}{|c|c|c|c|}
\hline & Bilirubin & Creatinine & $\mathrm{PT} / \mathrm{INR}$ \\
\hline \multicolumn{4}{|l|}{ Laboratory 1} \\
\hline Instrument & Vitros FS 5.1 (Ortho) & Vitros FS 5.1 (Ortho) & Compact/Star (Roche) \\
\hline Method & Colorimetric (DPD) & Enzymatic & Optical clot detection \\
\hline Thromboplastin & - & - & Innovin (DB) \\
\hline \multicolumn{4}{|l|}{ Laboratory 2} \\
\hline Instrument & Hitachi 917 (Roche) & Hitachi 917 (Roche) & Stago STA-R \\
\hline Method & Colorimetric (DPD) & Jaffe colorimetric & Optical clot detection \\
\hline Thromboplastin & - & - & Neoplastine $\mathrm{Cl}_{+}$ \\
\hline \multicolumn{4}{|l|}{ Laboratory 3} \\
\hline Instrument & Roche 018 & Roche 690 & Dade Behring CA-1500 \\
\hline Method & Colorimetric (DPD) & Jaffe colorimetric & Optical clot detection \\
\hline Thromboplastin & - & - & Innovin (DB) \\
\hline \multicolumn{4}{|l|}{ Laboratory 4} \\
\hline Instrument & Modular P (Roche) & Modular P (Roche) & Dade Behring BCS \\
\hline Method & Colorimetric (DPD) & Jaffe method & Optical clot detection \\
\hline Thromboplastin & - & - & Thromborel S (DB) \\
\hline \multicolumn{4}{|l|}{ Laboratory 5} \\
\hline Instrument & Beckman Coulter & Beckman Coulter & CA 7000 (Sysmex) \\
\hline Method & Colorimetric (DPD) & Jaffe colorimetric & Optical clot detection \\
\hline Thromboplastin & - & - & Innovine (DB) \\
\hline \multicolumn{4}{|l|}{ Laboratory 6} \\
\hline Instrument & Modular P (Roche) & Modular P (Roche) & Dade Behring BCS \\
\hline Method & Colorimetric (DPD) & Jaffe Method & Optical clot detection \\
\hline Thromboplastin & - & - & Thromborel S (DB) \\
\hline
\end{tabular}

DB, Dade Behring; DPD, n-diethyl-p-phenylene diamine. 
Schouten et al.

Table 2. INR (mean $\pm \mathrm{SD}$ ) obtained in patients with Child $\mathrm{A}, \mathrm{B}$, and $\mathrm{C}$ cirrhosis, control patients, and patient taking oral anticoagulants in the six laboratories

\begin{tabular}{|c|c|c|c|c|c|}
\hline & INR Control patients & INR Patients on $\mathrm{OA}$ & INR Child A patients & INR Child B patients & INR Child C patients \\
\hline \multicolumn{6}{|l|}{ Laboratory 1} \\
\hline Mean \pm SD & $1.02 \pm 0.06$ & $2.59 \pm 0.65$ & $1.08 \pm 0.08$ & $1.22 \pm 0.14$ & $1.45 \pm 0.32$ \\
\hline \multicolumn{6}{|l|}{ Laboratory 2} \\
\hline Mean \pm SD & $1.16 \pm 0.13$ & $2.80 \pm 0.51$ & $1.25 \pm 0.13$ & $1.45 \pm 0.15$ & $1.87 \pm 0.58$ \\
\hline \multicolumn{6}{|l|}{ Laboratory 3} \\
\hline Mean \pm SD & $1.03 \pm 0.56$ & $2.61 \pm 0.67$ & $1.09 \pm 0.87$ & $1.22 \pm 0.15$ & $1.46 \pm 0.31$ \\
\hline \multicolumn{6}{|l|}{ Laboratory 4} \\
\hline Mean \pm SD & $1.03 \pm 0.10$ & $2.55 \pm 0.45$ & $1.12 \pm 0.16$ & $1.27 \pm 0.21$ & $1.63 \pm 0.49$ \\
\hline \multicolumn{6}{|l|}{ Laboratory 5} \\
\hline Mean \pm SD & $0.98 \pm 0.07$ & $2.38 \pm 0.52$ & $1.06 \pm 0.01$ & $1.20 \pm 0.16$ & $1.42 \pm 0.31$ \\
\hline \multicolumn{6}{|l|}{ Laboratory 6} \\
\hline Mean \pm SD & $1.06 \pm 0.08$ & $2.76 \pm 0.56$ & $1.16 \pm 0.15$ & $1.37 \pm 0.23$ & $1.80 \pm 0.58$ \\
\hline
\end{tabular}

$\mathrm{OA}$, oral anticoagulants; INR, international normalized ratio; SD, standard deviation.

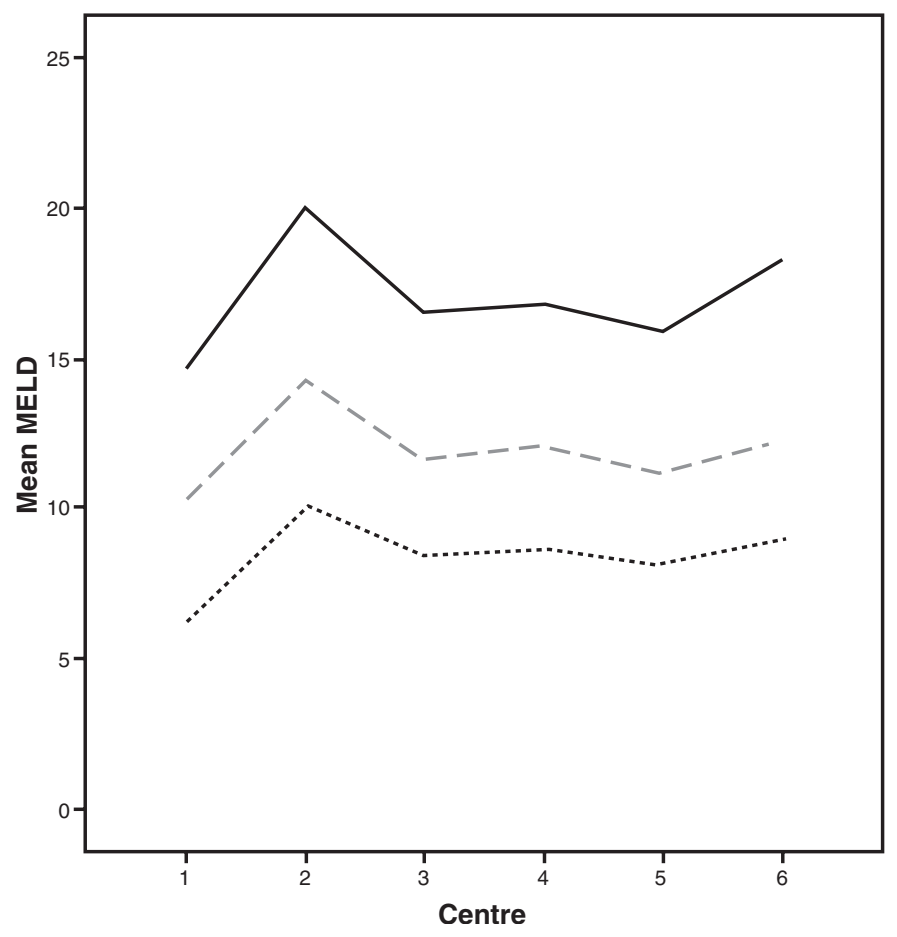

Patient group
..... Child A
-- Child B
- Child C

differences are present not only for patients with liver cirrhosis but also for patients on OAs. Laboratories using the same thromboplastin show comparable INR values (laboratories 1, 3, and 5).

Statistical significant interlaboratory differences were present in patients with Child $\mathrm{A}$ and $\mathrm{C}$ liver cirrhosis, patients taking OAs, and healthy controls in mean creatinine according to the six laboratories. These differences occurred between laboratories using different methods but
Fig. 1. Interlaboratory differences in MELD score for patients with Child A, $\mathrm{B}$, and $\mathrm{C}$ cirrhosis. MELD score in center 1 is statistically different from centers 2, 4, and 6. MELD score in centers 2 and 6 is statistically different from centers 1, 3, 4, and 5. MELD score in center 3 is statistically different from centers 2 and 6. MELD score in center 4 statistically different from centers 1,2 , and 6. MELD score in center 5 is statistically different from centers 1,3 , and 5 . These differences count for patients with Child B and Child $\mathrm{C}$ cirrhosis. surprisingly also between laboratories using comparable methods (laboratories 4 and 6). The highest observed difference between two laboratories was $0.128 \mathrm{mg} / \mathrm{dL}$ (laboratories 2 and 6).

Statistical significant differences in bilirubin were only present for patients with Child $\mathrm{A}$ and $\mathrm{C}$ cirrhosis (elevated bilirubin) and patients taking OAs. The highest interlaboratory differences were present between laboratory 1 and laboratory 2 (difference in mean bilirubin $1.65 \mathrm{mg} / \mathrm{dL}$ ). Laboratories using 
comparable methods and instruments did not yield differences in bilirubin (laboratories 4 and 6).

Mean MELD score of cirrhotic patients with Child A, B, and $\mathrm{C}$ liver cirrhosis for the six different laboratories along with the standard deviation of the cohort is shown in Table 3. Fig. 1 depicts the calculated mean MELD score for each laboratory according to Child classification. Statistically significant interlaboratory differences are present between the six centers for all patients with liver disease. The highest observed mean difference in MELD score in patients with cirrhosis was 5.76 (between centers 1 and 2). Statistically significant MELD score differences were also present in patients with Child A and Child B cirrhosis. Laboratories using comparable instruments, methods, and thromboplastins report comparable MELD scores (laboratories 1, 3, and 5) in patients with Child C and B cirrhosis.

Fig. 2 and Table 4 show results when selecting all patients with a MELD score higher than 17 in center 2. Highest interlaboratory differences in MELD score were reported between center 1 and 2 as well (difference mean MELD: 6.45) for patients with cirrhosis.

The univariate Cox proportional hazards model shows that patients with the highest MELD score had the highest probability of receiving a liver graft
Fig. 2. MELD (>17) in each center according to Child score. MELD score in center 1 is statistically different from centers 2, 4, and 6. MELD scores in centers 2 and 6 are statistically different from centers 1, 3, 4, and 5. MELD score in center 3 is statistically different from centers 2 and 6. MELD score in center 4 is statistically different from centers 1, 2, and 6. MELD in center 5 is statistically different from centers 1,3 , and 5. These differences count for patients with Child B and Child C cirrhosis.

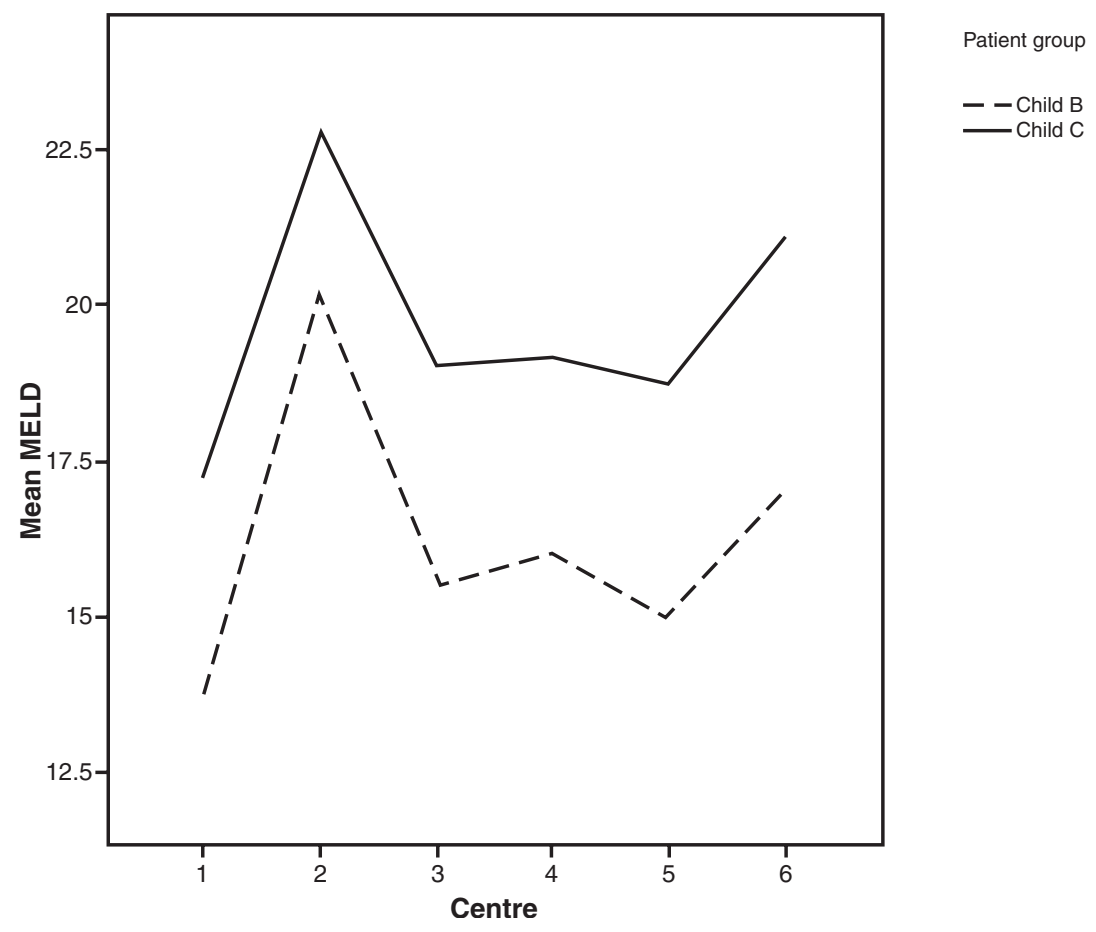

Table 3. MELD score (mean \pm SD) obtained in the six laboratories in Child A, B, and C cirrhosis

\begin{tabular}{|c|c|c|c|}
\hline & MELD score Child A & MELD score Child B & MELD score Child C \\
\hline \multicolumn{4}{|l|}{ Laboratory 1} \\
\hline Mean \pm SD & $5.57 \pm 4.21$ & $10 \pm 3.69$ & $13.48 \pm 5.75$ \\
\hline \multicolumn{4}{|l|}{ Laboratory 2} \\
\hline Mean \pm SD & $10.00 \pm 2.97$ & $13.31 \pm 2.56$ & $19.24 \pm 5.56$ \\
\hline \multicolumn{4}{|l|}{ Laboratory 3} \\
\hline Mean \pm SD & $8.46 \pm 2.94$ & $11.61 \pm 3.21$ & $16.5 \pm 6.46$ \\
\hline \multicolumn{4}{|l|}{ Laboratory 4} \\
\hline Mean \pm SD & $8.64 \pm 3.22$ & $11.73 \pm 3.38$ & $16.84 \pm 5.50$ \\
\hline \multicolumn{4}{|l|}{ Laboratory 5} \\
\hline Mean \pm SD & $8.07 \pm 3.15$ & $10.89 \pm 3.03$ & $15.08 \pm 5.40$ \\
\hline \multicolumn{4}{|l|}{ Laboratory 6} \\
\hline Mean \pm SD & $8.92 \pm 3.26$ & $12.05 \pm 3.34$ & $17.64 \pm 6.09$ \\
\hline
\end{tabular}


Schouten et al.

Table 4. MELD score (mean \pm SD) obtained in patients with MELD score above 17 in the six different laboratories

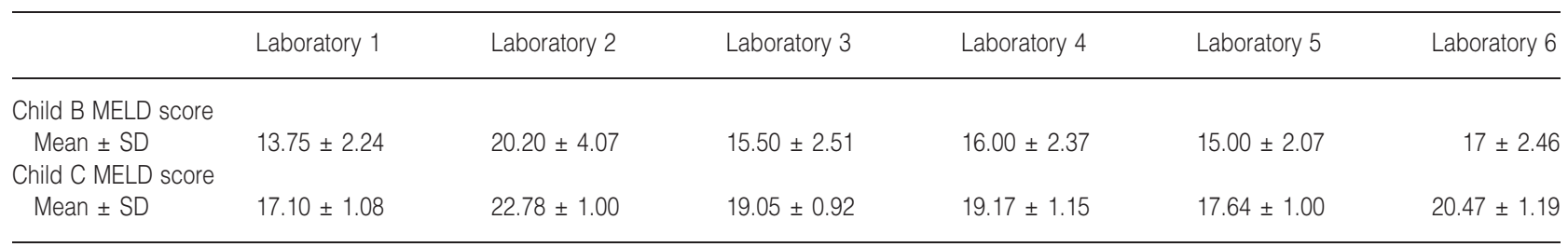

MELD score, model for end-stage liver disease; SD, standard deviation.

as reflected by the highest hazard ratios. The unadjusted hazard ratio for receiving a liver transplant is significantly different $(\mathrm{p}<0.0001)$ between group 2 and group 4 (group 2: 19-24; group 4: > 30) (Fig. 3, Table 5). MELD score remains independently associated with the probability of receiving a liver graft after adjusting for sex and recipient age in a multivariate model (Fig. 4).

\section{Discussion}

In this large multicenter prospective study, we demonstrated statistically significant interlaboratory differences in all three parameters of the MELD formula because of differences in instruments, methods of measurement, and used thromboplastins. In addition, we demonstrated for the first time that these interlaboratory differences, in patients with high MELD scores, might lead to laboratory-dependent influence on the probability of receiving a liver transplant.

Considering INR, our study confirmed aforementioned results by demonstrating interlaboratory INR differences between the participating centers $(18,20,22)$. Centers using thromboplastins with comparable ISI and different instruments (centers 1, 3, and 5) did not yield significant differences in INR, posing used thromboplastin above instrument for the interlaboratory differences in INR. Centers 2 and 6 using thromboplas-

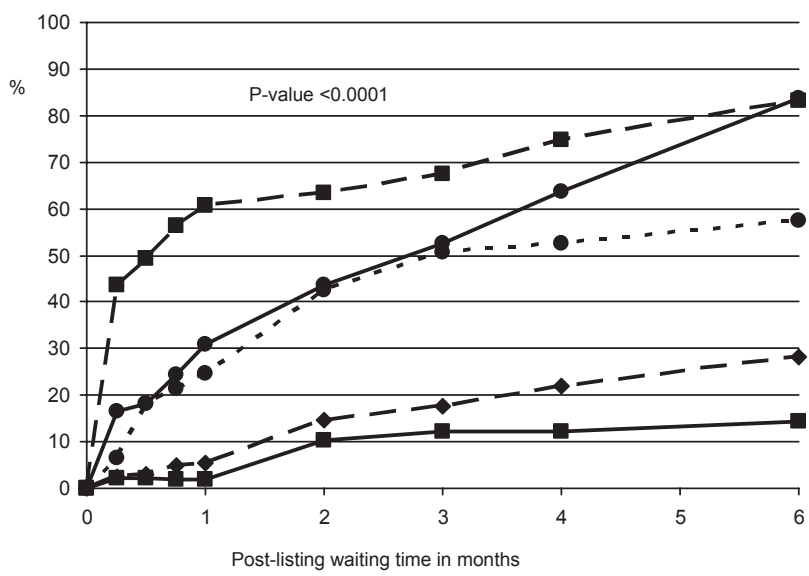

tins with a higher ISI (the thromboplastins have an ISI range from 1 to 3 , the lower number indicating a more sensitive thromboplastin) consequently display the highest INR in the group of patients with liver cirrhosis (Child A, B, and C). The centers reporting the lowest INR values used a very sensitive recombinant human thromboplastin. Although less clinically relevant, the differences in INR were present not only in patients with low INR (Child A and B patients) but also in patients with Child $\mathrm{C}$, meaning that the interlaboratory difference of INR is a problem covering the whole INR range. The previously reported differences in INR are comparable with the INR variation in our study $(25-40 \%)(18,22)$.

Obviously, the implementation of INR in the MELD score is scientifically incorrect, because no studies were performed assessing the value of this test developed for patients on OAs in patients with

Table 5. The unadjusted hazard ratios and $95 \%$ confidence intervals (Cl) for the probability of receiving a post-mortem liver transplantation according to MELD score groups

\begin{tabular}{lcc}
\hline Variable & Hazard ratios & $95 \% \mathrm{Cl}$ \\
\hline MELD 11-18 & 1.62 & $0.87-3.03$ \\
MELD 19-24 & 4.35 & $2.26-8.38$ \\
MELD 25-29 & 12.13 & $6.41-22.96$ \\
MELD $>$ 30 & 18.65 & $9.89-35.15$ \\
\hline
\end{tabular}

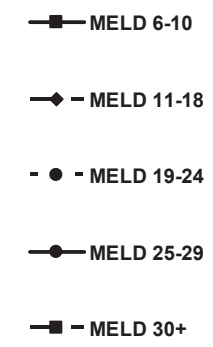

Fig. 3. Probability of receiving a postmortem liver transplant for all elective liver transplantation candidates with cirrhosis as primary disease stratified by match MELD (period of registration from December 2006 till June 2007). 
Fig. 4. Probability of receiving a postmortem liver transplant. Adjusted hazard ratios $(95 \%$ confidence intervals).

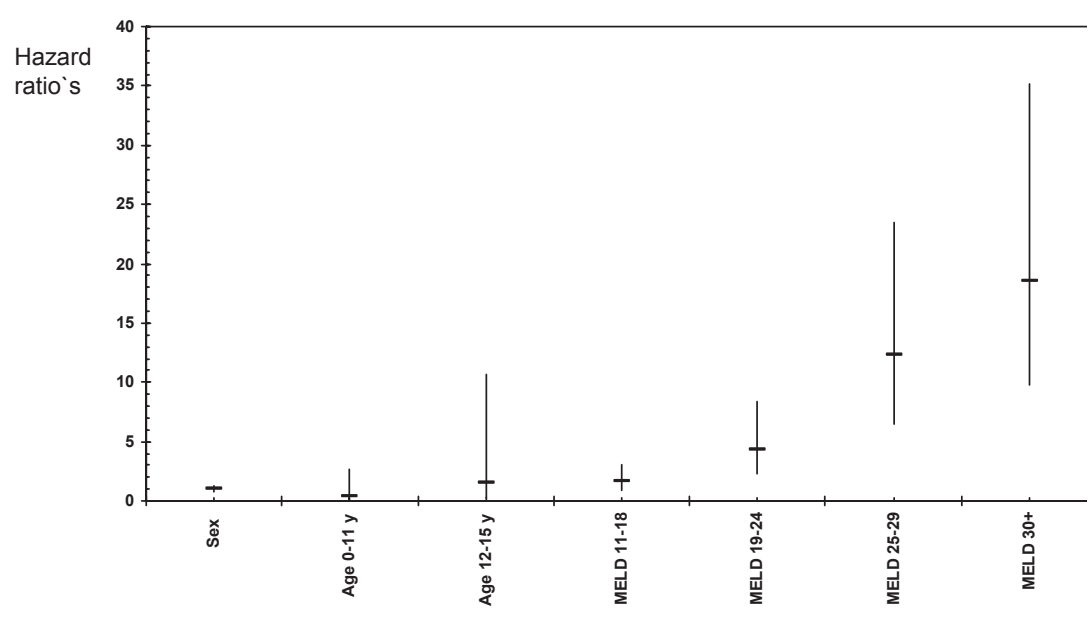

liver failure. The explanation for the wide variation in INR in liver patients compared with patients on $\mathrm{OA}$ is that coagulation disorders in patients with liver disease differ considerable from disorders in patients taking OAs. In the latter group, the inhibition of vitamin K-dependent gamma-carboxylation of coagulation factors II, VII, IX, and X causes a decrease in these factors, whereas in patients with liver failure leads to the decreased production of nearly all factors (except factor VIII).

Surprisingly, interlaboratory variation does not exclusively occur in patients with liver disease but also in patients taking OA. Considering that the INR originally was designed for standardization of the PT to monitor patients on treatment with OAs, these results are alarming, but not surprising. Interlaboratory INR differences in patients on OA were reported before (24).

The interlaboratory differences in INR determination cannot be attributed to freezing of the samples. Because of the potential for deterioration in function and concentration of clotting factors, the INR seems the most vulnerable parameter compared with creatinine and bilirubin. However, numerous studies have demonstrated the lack of differences in INR between frozen and freshly assayed samples $(25,26)$. The effect of freezing the samples is considered negligible.

Contrasting interlaboratory differences in INR, Trotter et al. did not demonstrate significant differences in bilirubin and creatinine. Conversely, differences in creatinine were reported in patients with and without liver disease (partly due to negative interference of bilirubin elevation with creatinine measurements) (19-21). Our study results confirm these data. We could demonstrate statistically significant differences in creatinine between the six laboratories. Laboratories using the same methods and instruments demonstrated only differences in patients with Child $\mathrm{C}$ cirrhosis (high bilirubin), not in patients with Child $\mathrm{A}$ and $\mathrm{B}$ cirrhosis. These differences were smaller compared with differences in INR; probably, the differences would be more pronounced in patients with higher creatinine levels.

Up to now, no laboratory-dependent differences were demonstrated for bilirubin, and our study is the first to reveal statistical significant differences (up to $26 \%$ ) in patients with severe and mild liver cirrhosis (Child $\mathrm{C}$ and $\mathrm{A}$ ). No differences in methods of bilirubin analyses were noted; the differences can only be explained by the use of different instruments. Remarkably, the highest bilirubin was noted in the laboratories using the thromboplastin with highest ISI. We are, however, not aware of any influence of INR on bilirubin determination. In line with interlaboratory differences in INR, the potential freezing effect of the creatinine and bilirubin samples on the observed interlaboratory differences can be considered minimal. Both assays are stable when frozen at $-20^{\circ}$ (27). Therefore, we do not believe that sample freezing could be responsible for the differences in INR, creatinine, and bilirubin values between the clinical laboratories in this study.

Obviously, these interlaboratory differences in INR, bilirubin, and creatinine yield to differences in MELD score. Centers 2 and 6 have significant higher MELD scores for patient with Child A, B, and $\mathrm{C}$ liver cirrhosis compared with centers 1,3 , and 5. The highest observed difference in mean MELD score between two centers is 5.76. When selecting only patients with a MELD score higher than 17 (in center 2), the highest observed difference in mean MELD score is 6.45 , demonstrating that the laboratory-dependent MELD score differences at least persist in patients with higher MELD scores. 
The clinical relevance of these differences was, up to now, a subject of debate. Recently, Ravaioli et al. (23) showed higher dropout in patients on the waiting list for liver transplantation in one center compared with another probably due to interlaboratory differences. However, statistical significant differences in the probability of organ allocation between patients with different MELD scores have yet not been reported. We are the first to demonstrate the clinical importance of the laboratorydependent MELD score variation in the probability of organ allocation. Our analyses (based on ET results of the first six months of MELD implementation) confirmed the presumption that the highest hazard ratios for receiving a liver transplant would be observed in patients with MELD score above 30, decreasing in the groups with lower MELD scores. Cox proportional hazards model showed the significant association between the MELD score level and the probability of receiving an organ (patients with higher MELD scores have significant higher probability of receiving a donor liver) remaining after adjusting for sex and age in multivariate analyses. The probability of organ allocation differs statistically significant between patients with MELD score 19-24 and patients with MELD score $>30$ (minimum difference of six MELD points). Regarding that the laboratoryinduced differences in mean MELD score between center 1 and center 2 were 6.12 MELD points, we can conclude that a patient in center 2 with a MELD score of 30 will have a significant lower probability of receiving an organ in center 1 having a MELD score of 24. The patients with cirrhosis included in this study were on the waiting list for liver transplantation during the first six months of MELD implementation (time period of hazard ratio's calculation). Hereby, the results of this study demonstrate for the first time in literature that this laboratory/center-dependent disparity in MELD scores might influence the probability of organ allocation. This observation implicates that patients having MELD score parameters determined in a center with laboratory methods leading to higher MELD score calculation (center 2) can be favoured compared with patients having MELD score parameters determined in a center using methods providing lower MELD score (center 1). Obviously, this observation questions the "sickest first" policy of MELD score implementation.

Stemming from the results of this study, it can be concluded that solutions to eradicate the problem of the interlaboratory differences in MELD score are warranted. Given the high impact of INR in the MELD formula and the significant laboratory variation in this parameter, the main research should be focused on the variation of this parameter. In 2007, two studies were carried out independently to resolve this in patients with liver disease. These studies prove that an alternative calibration model modified from that recommended by the World Health Organisation for patients on OAs may be feasible for patients on OAs $(28,29)$. These results are promising, especially because a recent paper of Tripodi et al. demonstrated that the INR liver calibration for INR calibration also works for easy-to-run whole blood coagulation monitors. This implicates that once the monitors are calibrated by the manufacturer in terms of INR liver, it is ready to use in liver units (30). However, additional study is needed particularly to investigate whether these differences in INR liver would not re-emerge after transporting the samples to different laboratories from the one that standardized this liver-INR. We demonstrated that laboratories using comparable methods, techniques, and instruments yield similar MELD scores, meaning that the centralization of determination of the different MELD parameters in a defined laboratory or standardization of methods, techniques, and instrument between laboratories in liver transplant centers are alternatives to avoid interlaboratory differences. As patients on the waiting list for liver transplantation sometimes have their laboratory determined in different laboratories, this option is difficult to realize.

Alternative solutions might be to either use the PT expressed as a percentage against "normal patients" as a measure of coagulant activity of the liver, or to use another "single" clotting factor that is representative of liver function. Single clotting factor tests are technicality straightforward and have smaller variability between laboratories. As to the choice of representative clotting factors, a case can be made for factor V or antithrombin III as the most suitable markers; however, this has to be based upon large prognostic studies. Promising results have been reported regarding PT expressed as a percentage, and Robert et al. (31) demonstrated that its use eliminates most of the thromboplastin-induced PT variability in patients with liver disease.

In conclusion, this large prospective multicenter study confirms the interlaboratory differences in MELD score because of laboratory dependency in three different parameters of the MELD score. We demonstrated for the first time that these interlaboratory differences might lead to statistically significant center-dependent differences in the probability of receiving a transplant in patients with a MELD score above 17. Clearly, having demonstrated the clinical relevance of the center-dependent influence 
on MELD score calculation, instant measures should be undertaken to standardize the determination of INR, creatinine, and bilirubin between different liver transplant centers to avoid laboratory-dependent allocation and to improve this model of liver allocation.

\section{References}

1. Wiesner R, Edwards E, Freeman R et al. Model for end-stage liver disease (MELD) and allocation of donor livers. Gastroenterology 2003: 124: 91.

2. Malinchoc M, Kamath PS, Gordon FD, Peine CJ, RANK J, TER BORG PC. A model to predict poor survival in patients undergoing transjugular intrahepatic portosystemic shunts. Hepatology (Baltimore, MD) 2000: 31: 864.

3. Said A, Williams J, Holden J et al. Model for end stage liver disease score predicts mortality across a broad spectrum of liver disease. J Hepatol 2004: 40: 897.

4. Heuman DM, Mihas A. Utility of the MELD score for assessing 3-month survival in patients with liver cirrhosis: one more positive answer. Gastroenterology 2003: 125: 992. author reply 4-5.

5. Gheorghe L, Iacob S, Iacob R, Gheorghe C, Popescu I. Variation of the MELD score as a predictor of death on the waiting list for liver transplantation. J Gastrointestin Liver Dis 2007: 16: 267.

6. Ferraz-Neto BH, Hidalgo R, Thome T et al. Analysis of Model for End-Stage Liver Disease (MELD) score in a liver transplantation waiting list. Transpl Proc 2007: 39: 2511.

7. Botta F, Giannini E, Romagnoli P et al. MELD scoring system is useful for predicting prognosis in patients with liver cirrhosis and is correlated with residual liver function: a European study. Gut 2003: 52: 134.

8. Freeman RB, Wiesner RH, Edwards E, Harper A, Merion R, Wolfe R. Results of the first year of the new liver allocation plan. Liver Transpl 2004: 10: 7.

9. BROWN RS JR, LAKE JR. The survival impact of liver transplantation in the MELD era, and the future for organ allocation and distribution. Am J Transplant 2005: 5: 203.

10. Kamath PS, Kim WR. The model for end-stage liver disease (MELD). Hepatology (Baltimore, MD) 2007: 45: 797.

11. Gish RG. Do we need to MEND the MELD? Liver Transpl 2007: 13: 486.

12. Freeman RB JR. MELD and liver allocation: continuous quality improvement. Hepatology (Baltimore, MD) 2004: 40: 787.

13. EVERSON GT. MELD: the answer or just more questions? Gastroenterology 2003: 124: 251.

14. Durand F, Valla D. Assessment of the prognosis of cirrhosis: Child-Pugh versus MELD. J Hepatol 2005: 42(Suppl 1): S100.

15. Cholongitas E, Senzolo M, Triantos C, Samonakis D, PATCh D, Burroughs AK. MELD is not enough - enough of MELD? J Hepatol 2005: 42: 475. author reply 8-9.
16. Cholongitas E, Marelli L, Shusang V et al. A systematic review of the performance of the model for end-stage liver disease (MELD) in the setting of liver transplantation. Liver Transpl 2006: 12: 1049.

17. Burroughs AK, Marelli L, Cholongitas E et al. Towards a better liver transplant allocation system. Liver Transpl 2007: 13: 935. author reply 7.

18. Trotter JF, Brimhall B, Arjal R, Phillips C. Specific laboratory methodologies achieve higher model for endstage liver disease (MELD) scores for patients listed for liver transplantation. Liver Transpl 2004: 10: 995.

19. Kerry ACE, Marelli L, Goodier D, Nair D, Thomas M, Burroughs A. MELD score and creatinine measurements. Clin Chim Acta 2005: 355: A153.

20. Cholongitas E, Marelli L, Kerry A et al. Different methods of creatinine measurement significantly affect MELD scores. Liver Transpl 2007: 13: 523.

21. Bzoweu NHV, Sebanc H, Bonacini M, Rabkin J, Doo E. MELD underestimated unless enzymatic creatinie used. J Hepatol 2004: 40(Suppl 2): 40A.

22. Lisman T, van Leeuwen $\mathbf{Y}$, Adelmeijer $\mathbf{J}$ et al. Interlaboratory variability in assessment of the model of endstage liver disease score. Liver Int 2008: 28: 1344.

23. Ravaioli M, Masetti M, Ridolfi L et al. Laboratory test variability and model for end-stage liver disease score calculation: effect on liver allocation and proposal for adjustment. Transplantation 2007: 83: 919.

24. Poller L, Keown M, Ibrahim $\mathrm{S}$ et al. Comparison of local International Sensitivity Index calibration and 'Direct INR' methods in correction of locally reported International Normalized Ratios: an international study. J Thromb Haemost 2007: 5: 1002.

25. van den BesselaAr AM, Houbouyan-Reveillard LL, Aillaud MF et al. Multicenter evaluation of lyophilized and deep-frozen plasmas for assignment of the International Normalized Ratio. Thromb Haemost 1999: 82: 1451.

26. Grau E, Tenias JM, Olaso MA et al. Monitoring oral anticoagulant treatment from plasma stored for up to 48 hours and frozen plasma. Haematologica 1999: 84: 633.

27. Young DS, editor. Effects of Preanalytical Variables on Clinical Laboratory Tests, 2nd edn, AACC Press: Washington, DC, 1997.

28. Tripodi A, Chantarangkul V, Primignani $\mathrm{M}$ et al. The international normalized ratio calibrated for cirrhosis (INR(liver)) normalizes prothrombin time results for model for end-stage liver disease calculation. Hepatology (Baltimore, MD) 2007: 46: 520 .

29. Bellest L, Eschwege V, Poupon R, Chazouilleres O, RoBert A. A modified international normalized ratio as an effective way of prothrombin time standardization in hepatology. Hepatology (Baltimore, MD) 2007: 46: 528.

30. Tripodi A, Chantarangkul V, Primignani $M$ et al. Point-of-care coagulation monitors calibrated for the international normalized ratio for cirrhosis (INRliver) can help to implement the INRliver for the calculation of the MELD score. J Hepatol 2009: 51: 288.

31. Robert A, Chazoullleres O. Prothrombin time in liver failure: time, ratio, activity percentage, or international normalized ratio? Hepatology (Baltimore, MD) 1996: 24: 1392. 